\title{
Non-Uniform Reductions
}

\author{
Harry Buhrman • Benjamin Hescott • \\ Steven Homer • Leen Torenvliet
}

Published online: 9 January 2009

(C) The Author(s) 2009. This article is published with open access at Springerlink.com

\begin{abstract}
We study properties of non-uniform reductions and related completeness notions. We strengthen several results of Hitchcock and Pavan (ICALP (1), Lecture Notes in Computer Science, vol. 4051, pp. 465-476, Springer, 2006) and give a tradeoff between the amount of advice needed for a reduction and its honesty on NEXP. We construct an oracle relative to which this trade-off is optimal. We show, in a more systematic study of non-uniform reductions, among other things that non-uniformity can be removed at the cost of more queries. In line with Post's program for complexity theory (Buhrman and Torenvliet in Bulletin of the EATCS 85, pp. 41-51, 2005) we connect such 'uniformization' properties to the separation of complexity classes.
\end{abstract}

Keywords Non-uniform reductions $\cdot$ Reductions with advice $\cdot$ Non-uniform complexity $\cdot$ NEXP complete set $\cdot$ EXP complete set $\cdot$ NP complete set

\section{Introduction}

Reductions and completeness are two of the original concepts in complexity theory and form part of the core of the field to this day. Determining whether a problem is

H. Buhrman

CWI, Kruislaan 409, 1098 SJ Amsterdam, The Netherlands

e-mail: buhrman@cwi.nl

B. Hescott $(\bowtie)$

Computer Science Department, Tufts University, 161 College Ave, Medford, MA 02155, USA

e-mail: hescott@cs.tufts.edu

S. Homer

Computer Science Department, Boston University, 111 Cummington St, Boston, MA 02215, USA

L. Torenvliet

ILLC, Plantage Muidergracht 24, Amsterdam, The Netherlands

e-mail: leen@science.uva.nl 
complete for a particular class is central in determining the computational complexity of that problem. In a broader perspective, questions of whether different types of reductions are the same or different, and whether completeness notions induced by these reductions are the same or different have been assiduously explored, e.g. $[12,13,27,30]$. Answers to these questions are related to the central open problems of the field. In some cases, inequality of reductions on certain complexity classes implies inequality of complexity classes. In others, the collapse of degrees to an isomorphism type under some notion of reduction also yields the inequality of classes [2, 3 , $7,16,19,23,25,31]$. This makes the properties of reductions, for example whether they are length-increasing, 1-1, etc. important objects of study. Finally, certain properties of complete sets in different complexity classes might have implications for the of equality of these classes $[5,10,15]$.

The lion's share of investigations of reductions has been into uniform reductions. Now, non-uniformity has entered the realm of the reduction. As with the definitions of non-uniform complexity classes, by means of advice classes, $(\mathrm{P} /$ poly and families of circuits of a certain size), one can define reductions that are computable by means of additional advice or by polynomial size circuits.

Allender et al. [4] have shown that the set $R$ of Kolmogorov incompressible strings, with respect to exponential time Kolmogorov complexity, is complete for EXP with respect to polynomial time (truth-table) reductions that have a polynomial amount of advice. Moreover, the advice is indispensable: $R$ is not complete with respect to uniform Turing reductions [8]. For a strengthening of this see the thesis of Ronneburger [29].

Agrawal [1], while studying the isomorphism conjecture [7] for NP complete sets, used non-uniform reductions with advice. He showed, under the assumption that a certain type of one-way function exists, that all many-one complete sets for NP are 1-1 and length increasing complete for reductions that use some amount of nonuniform advice.

Hitchcock and Pavan [22] show, under a different assumption, namely that NP is not small (does not have resource bounded measure zero), that every many-one complete set for NP is length increasing complete with reductions that use a polynomial amount of advice. Moreover they also show that for NEXP the many-one complete sets are length increasing complete with reductions that use a polynomial amount of advice.

In this paper we improve the results of Hitchcock and Pavan. In particular, we show, under the weaker assumption that there exists a DTIME $\left(2^{n^{c}}\right)$ bi-immune set in $\mathrm{NP}$, that one bit of advice suffices to show that many-one complete sets are length increasing complete. ${ }^{1}$ We also improve their results for NEXP. We again reduce the length of the advice needed to make the reductions length increasing and show that our result is optimal relative to some oracle. In particular, this yields an oracle where the many-one complete sets for NEXP are not (uniformly) length increasing complete, in fact they are only exponentially honest, matching the best known result due

\footnotetext{
${ }^{1}$ Technically we show something slightly weaker. We show that the many-one reduction is either length increasing or accepts/rejects without querying a string. The result does hold for 1-tt reductions.
} 
to Ganesan and Homer [17]. This shows that non-relativizing techniques are needed to settle this question.

Another structural property known for EXP [20] and for NEXP [14] is the following: Every 1-truth-table complete set is many-one complete. We show that, under the hypothesis that NP contains a set that is $\operatorname{NTIME}\left(2^{n^{c}}\right) \cap \operatorname{co}-\operatorname{NTIME}\left(2^{n^{c}}\right)$ bi-immune, every 1-truth-table complete set for NP is many-one complete with 1 bit of advice. ${ }^{2}$ By extending an earlier result of [9], we construct an oracle world where such a biimmune set exists in NP. This result relates to the work of Glasser et al. [18]. They show under the assumption that NP differs from coNP at every length that 1-truthtable complete sets for NP are also many-one complete with polynomial advice.

The results above warrant a more systematic investigation into non-uniform reductions. In particular, is the amount of advice needed for the non-uniform reductions above optimal? In general, when does advice yield additional power?

In the second part of this paper we begin such a study. We first show that for EXP constant query reductions that have advice are strictly more powerful than their uniform counterparts. For example we show that the $\leq_{m}^{p / 1}$-complete degree and the $\leq_{2 \mathrm{tt}}^{p}$-complete degree for EXP are incomparable. Analogous to uniform reductions, we show that complete sets for EXP with respect to many-one reductions that use $c$ bits of advice are 1-1 and length increasing complete with $c$ bits of advice. These results require new techniques as the original ideas for uniform reductions cannot be used directly.

Second, we show that non-uniformity can be removed at the price of more queries. In particular we show that sets that are many-one complete with respect to reductions that use $O(\log n)$ bits of advice are Turing-complete with respect to uniform reductions for EXP. For the delta levels of the polynomial hierarchy we show that truthtable reductions suffice in order to 'uniformize' the reduction. Here we use ideas and techniques from the paper that studies the auto-reducibility of complete sets [15]. We have the added bonus that these theorems do not relativize and hope that these results could be used in a nonrelativizing proof that separates complexity classes. In particular it follows from our results that solving the question of whether many-one complete sets with 1 bit of advice are uniformly truth-table complete will separate complexity classes (EXP from EXPSPACE or PH from EXP). For details see Theorems 35 and 34. Hence understanding questions like these has deep implications for complexity theory.

\section{Notation}

We adopt the standard definitions and notations for well-known complexity classes, and other notions of computational complexity, as can be found, e.g., in [6] and [24]. We use the standard paring function denoted $\langle a, b\rangle$ for binary strings $a, b$, where $|\langle a, b\rangle|=2|a|+|b|+2$. Let $\pi_{i}(q)$ denote the $i$ th projection of the $k$-tuple $q$, where $i \leq k$. We use the standard definitions for polynomial time reductions, i.e., we say

\footnotetext{
${ }^{2}$ Again we technically prove this for the weaker version of many-one reduction that can also accept/reject without producing a query.
} 
$A$ many-one reduces to set $B$, denoted $A \leq_{m}^{p} B$, if there is a polynomial time computable function $f$ where $x \in A \leftrightarrow f(x) \in B$. The reduction is considered a 1-1 reduction, $\leq_{1-1}^{p}$, if in addition the function $f$ is one to one. A many-one reduction is length increasing, $\leq_{m, \mathrm{li}}^{p}$, if in addition, $\forall x,|f(x)|>|x|$. A reduction is honest if there is a polynomial $p$, s.t. $\forall x, p(|f(x)|)>|x|$. We recall the notion of the extended many-one reduction: $A \leq_{\hat{m}}^{p} B$ if there exists a polynomial time Turing machine $M$ with an output tape, where on input $x, M$ does one of the following: $M$ outputs $f(x) \in \Sigma^{*}$ and $x \in A \leftrightarrow f(x) \in B, M$ outputs ACCEPT and $x \in A$, or $M$ outputs REJECT and $x \notin A$.

Polynomial time oracle machines are used to characterize both Turing and truthtable reductions. The query set of an oracle machine $M$ on input $x$ with oracle $A$ is denoted as $Q\left(M^{A}(x)\right)$. The notation $M^{A}(x)$ is also used as a notation for the outcome of the computation of machine $M$ on input $x$ with oracle $A$. This can be either accept/reject or a string $y$. In the latter case-the machine computes a function-we also use the notation $f^{A}(x)$. We assume enumerations $\left\{M_{i}\right\}_{i}\left(\left\{f_{i}\right\}_{i}\right)$ for all convenient classes of machines (functions). A polynomial reduction is nonuniform if it is in $P /$ poly, i.e. $A \leq_{m}^{P / p o l y} B$ if $\exists f \in \mathrm{FP}$ and $h \in$ poly where $A=\{x \mid f(x, h(|x|)) \in B\}$, here poly $=\{g|\forall n| g,(n) \mid \leq p(n)$ for some polynomial $p\}$. Many times the amount of advice is more or less restrictive, this restriction is on the function $h$ in the definition above. Namely, for one bit of advice the range of $h$ is $\{0,1\}$. We denote a one-bit nonuniform many one reduction by $\leq_{m}^{p / 1}$. In many complexity classes $K$ will stand for the generic complete set, e.g., $K^{A}=\left\{\langle i, x, k\rangle \mid M^{A}(x)\right.$ accepts in $\leq k$ steps $\}$ is the canonical complete set for EXP with oracle $A$. To avoid confusion we will subscript $K$ with the appropriate complexity class, e.g., $K_{\mathrm{EXP}} . M_{K}$ or $N_{K}$ will be the machine accepting this set for deterministic, or resp. nondeterministic complexity classes.

A reduction from set $A$ to $B$ is considered to be adaptive if the membership of a string $x \in A$ is decided by a polynomial-time Turing machine which has oracle access to $B$. Here the computation is allowed to adapt to membership queries to $B$, by basing future queries on previous answers. This reduction is denoted $A \leq_{T} B$ and is commonly referred to as a Turing reduction. It is natural to consider reductions that are computed with oracle access to the set $B$, but are not adaptive. We say $A$ reduces to $B$ nonadaptively, denoted, $A \leq_{\mathrm{tt}}^{p} B$ if there exist a polynomialtime function, $r$ and Turing machine $M$ where $r(x)=\left(q_{1}, q_{2}, \ldots, q_{m}\right)$ and $M(x$, $\left.\left[q_{1} \in B\right],\left[q_{2} \in B\right], \ldots,\left[q_{m} \in B\right]\right)$ accepts if and only if $x \in A$, these reductions are commonly referred to as truth table reductions.

In this work we consider the measure hypothesis on NP formulated by Lutz which states that NP does not have $p$-measure $0, \mu(\mathrm{NP}) \neq 0$. This is equivalent to assuming that there is no polynomial time martingale that succeeds on every language in NP. A martingale is a function $d: \Sigma^{*} \rightarrow[0, \infty)$ where $\left(\forall w \in \Sigma^{*}\right)[2 d(w)=$ $d(w 0)+d(w 1)]$. A martingale succeeds on language L if $\limsup _{n \rightarrow \infty} d\left(A^{\leq n}\right)=\infty$. Given a time bound $t(n)$ a language $\mathrm{L}$ is considered to be $t(n)$-random if no $O(t(n))$ martingale succeeds on L. Informally this relates to whether or not NP is a "large" subset of E.

The measure hypothesis on NP also implies the existence of a DTIME( $\left.2^{n^{\epsilon}}\right)$-biimmune set within NP [28]. A set is C-immune if it does not have any infinite subsets in $\mathrm{C}$, it is $\mathrm{C}$-bi-immune if it and its complement are both $\mathrm{C}$-immune. 


\section{Advice to Strengthen Reductions}

\subsection{Length Increasing Reductions}

Hitchcock and Pavan [22] show that some reductions can be made length increasing under the assumption that NP is not small. We improve upon the advice needed in their paper in the following theorem.

Theorem $1 \mu(\mathrm{NP}) \neq 0 \Longrightarrow$ Every $\leq_{m}^{p}$-complete set is $\leq_{m, \mathrm{li}}^{p / O(\log \log n)}$-complete.

Proof Choose $\epsilon>0$ and let $R$ be a $n^{1+\epsilon}$-random set in NP. Recall that this means no $O\left(n^{1+\epsilon}\right)$ time martingale succeeds on $R$. For every $n$, let $x_{0}^{n}, \ldots, x_{2 \log n+2}^{n}$ be the lexicographically first $2 \log n+3$ strings of length $n$. We claim that for all but finitely many lengths $n$, there is an $i, i \leq 2 \log n+2$ where $\left[R\left(0^{n}\right) \neq R\left(x_{i}\right)\right]$.

Assume not, then we have infinitely many lengths $n^{\prime}$ where $\forall i, i \leq 2 \log n^{\prime}+2$, $\left[R\left(0^{n^{\prime}}\right)=R\left(x_{i}\right)\right]$. Now consider the following betting strategy. First divide the capital so that at each length $n$ we have $\frac{1}{2 n^{2}}$ in capital. Recall, $\sum_{n=0}^{\infty} \frac{1}{2 n^{2}} \leq 1$. At every length $n$ bet evenly on $\left\{0^{n}\right\}$. Use the outcome of $R\left(0^{n}\right)$ to bet all or nothing on $x_{1}^{n}, \ldots, x_{2 \log n+2}^{n}$. If the length $n$ is one of the $n^{\prime}$ where $\left(\forall i \leq 2 \log n^{\prime}+2\right)\left[R\left(0^{n^{\prime}}\right)=\right.$ $\left.R\left(x_{i}\right)\right]$ then we make $\frac{2^{2 \log n^{\prime}+2}}{2 n^{\prime 2}}$ at this length as we double our capital for each $x_{1}^{n^{\prime}}, \ldots, x_{2 \log n^{\prime}+2}^{n^{\prime}}$. If $n$ is not one of these lengths, we bet until we loose our capital for that length, then we bet evenly until the next length. Since $\frac{2^{2 \log n^{\prime}+2}}{2 n^{\prime 2}}>1$ and there are infinitely many such $n^{\prime}$, this martingale succeeds on $R$, contradicting our assumption that $R$ is a $n^{1+\epsilon}$-random set.

To identify an index $i$ for which $R\left(0^{n}\right) \neq R\left(x_{i}\right)$ we need about $\log \log n$ bits. Let

$$
D=\{\langle x, y, \phi\rangle|| x|=| y|=| \phi \mid \wedge R(x)+R(y)+\operatorname{SAT}(\phi) \geq 2\} .
$$

Now let $A$ be a $\leq_{m}^{p}$-complete set in NP, and let $M$ calculate the reduction from $D$ to $A$.

Next we show that by combining a padded version of the reduction from SAT to $D$ and the reduction from $D$ to $A$ then gives a length increasing reduction from SAT to $A$ using $O(\log \log n)$ bits of advice - the advice is needed to give the length of the padded string which might be polynomially longer than the input.

Suppose that for every $k$ there are infinitely many $\langle x, y, \phi\rangle$ such that $|M(\langle x, y, \phi\rangle)|<|\langle x, y, \phi\rangle|^{1 / k}$. The set $A$ is in NP so it is DTIME( $\left(2^{n^{c}}\right)$-computable for some $c$. Hence for infinitely many $\langle x, y, \phi\rangle$ it is DTIME $\left(2^{n}\right)$ computable whether $\langle x, y, \phi\rangle$ is in $D$. This gives a betting strategy since $M(\langle x, y, \phi\rangle) \notin A$ implies $x \notin R$ or $y \notin R$ and $M(\langle x, y, \phi\rangle) \in A$ implies $x \in R$ or $y \in R$. Assume $x$ is lexicographically less than or equal to $y$. Given the outcome of $M(\langle x, y, \phi\rangle) \in A$ use $1 / 3 \mathrm{~d}$ of the capital on $x \in R$ and, if necessary, the remaining $2 / 3 \mathrm{~d}$ of the capital on $y \in R$. In either case the capital grows to $4 / 3$. So SAT $\leq_{m, \mathrm{li}}^{p / \log \log n} D \leq_{m \text {, honest }}^{p} A$ which proves the theorem.

With a weaker assumption but a less standard notion of reduction we arrive at an even stronger conclusion. 
Theorem $2(\forall c>0)\left[(\exists R \in \mathrm{NP})\left[R\right.\right.$ is $\operatorname{DTIME}\left(2^{n^{c}}\right)$-bi-immune $] \Longrightarrow\left[A\right.$ is $\leq_{\hat{m}}^{p}$ complete $\Rightarrow A$ is $\leq_{\hat{m}, l i}^{p / 1}$ complete $]$.

Proof Suppose that $A$ is in $\operatorname{NTIME}\left(n^{d}\right)$. Let

$$
D=\left\{\begin{array}{l}
\langle\phi, 0\rangle: \phi \in \mathrm{SAT} \vee 0^{|\phi|} \in R \\
\langle\phi, 1\rangle: \phi \in \mathrm{SAT} \wedge 0^{|\phi|} \in R
\end{array}\right\} .
$$

It is easy to see that $\mathrm{SAT} \leq_{m, \mathrm{li}}^{p / 1} D$. It therefore suffices to prove that $D \leq_{\hat{m} \text {,honest }}^{p} A$. Let $M$ be the reduction from $D$ to $A$. By definition of $D$.

$$
\begin{aligned}
& \phi \notin \mathrm{SAT} \quad \Longrightarrow \quad\left[0^{|\phi|} \in R \Leftrightarrow M(\langle\phi, 0\rangle) \in A\right], \\
& \phi \in \mathrm{SAT} \quad \Longrightarrow \quad\left[0^{|\phi|} \in R \Leftrightarrow M(\langle\phi, 1\rangle) \in A\right] .
\end{aligned}
$$

If $(\forall k)\left(\exists^{\infty} \phi\right)\left[|M(\langle\phi, b\rangle)|<|\langle\phi, b\rangle|^{\frac{1}{k}}\right]$, then $(\forall k)\left(\exists^{\infty} \phi\right)\left[|M(\langle\phi, 0\rangle)|<|\langle\phi, 0\rangle|^{\frac{1}{k}}\right]$ or $(\forall k)\left(\exists^{\infty} \phi\right)\left[|M(\langle\phi, 1\rangle)|<|\langle\phi, 1\rangle|^{\frac{1}{k}}\right]$.

Then by the DTIME $\left(2^{n^{c}}\right)$-bi-immunity of $R$ and the fact that $M(\langle\phi, b\rangle)$ in $A$ is $\operatorname{NTIME}\left(n^{d}\right)$ and hence DTIME $\left(2^{n^{d}}\right)$ computable if $|M(\langle\phi, b\rangle)|<|\phi, b|^{\frac{c}{d}}$ we can conclude for almost all of these $\phi$ in the first case that $\phi \in \mathrm{SAT}$; if $\phi \notin \mathrm{SAT}$, we could calculate $0^{|\phi|} \in R$ in DTIME $\left(2^{n}\right)$. Similarly, in the second case we know that $\phi \notin \mathrm{SAT}$. So, for a suitable $k$ whenever $|M(\langle\phi, b\rangle)|<|\langle\phi, b\rangle|^{1 / k}$, we can decide membership of $\phi$ in SAT in P. In the remaining cases $M$ is honest.

Hitchcock and Pavan [22] show that for nondeterministic exponential time, no assumption is needed to make the many-one complete sets length increasing complete via reductions that use a polynomial amount of advice. Next we will improve this result to $n-\log n$ bits of advice.

Theorem $3(\forall c>0)\left[A \leq_{m}^{p}-\right.$ complete for NEXP $\Longrightarrow A \leq_{m, 1 \mathrm{i}}^{p / n-c \log n}$ complete $]$.

Proof Let $A$ be any $\leq_{m}^{p}$ NEXP complete set and let $K$ be the standard 1-1, length increasing, paddable, many-one complete set computable in $\operatorname{NTIME}\left(2^{n}\right)$. Let $p$ : $\Sigma^{*} \times \Sigma^{*} \rightarrow \Sigma^{*}$ be a polynomial time padding function for $K$ where $\forall x \forall r \quad x \in K \leftrightarrow$ $p(x, r) \in K$, and let $f$ be a polynomial time 1-1 reduction from $K$ to $A$. Since $f$ and $p$ are both $1-1$, for every $n$ there is some $r_{n} \in \Sigma^{n+1}$ where $\left|f\left(p\left(x, r_{n}\right)\right)\right|>|x|$ for all $x \in \Sigma^{n}$. Let $r^{\prime}$ be $r_{n}$ with the last $c \log n$ bits removed. Now consider the function $p^{\prime}$ which takes $x, r^{\prime}$ as input and tries all possible $n$ bit strings, $r_{i}$, that are extensions consistent with $r^{\prime}$ and outputs $f\left(p\left(x, r_{i}\right)\right)$ for the first $r_{i}$ where $\left|f\left(p\left(x, r_{i}\right)\right)\right|>|x|$. We know that such an extension exists and that it will be polynomial in $n$ to find such an $r_{i}$. Let $g(x)=p^{\prime}\left(x, r^{\prime}\right)$ be the reduction from $K$ to $A$.

The same proof yields a trade-off between the amount of advice and the honesty of the reduction.

Theorem 4 For all $c, c>0$, the many-one complete sets for NEXP are $g(|x|)$-honest for reductions that use $g(|x|)-c \log n$ bits of advice. 
Corollary 5 [17] For any $c$, the many-one complete sets for NEXP are c $\log n$-honest complete.

Next we show that this amount of advice is optimal relative to some oracle. First we construct an oracle witnessing that NEXP has a many-one complete set which is not complete under length increasing many-one reductions. We then show how to adapt this oracle to witness that NEXP has a many-one complete set which is not complete under length increasing many-one reductions that use less than logarithmic advice.

Theorem 6 There exists an oracle witnessing that NEXP has a many one complete set that is not complete under length increasing reductions.

Proof We construct sets $A, C$, and $D$, such that $C=K_{\mathrm{NEXP}}^{A}-D$ is complete for $\mathrm{NEXP}^{A}$, but not via length-increasing reductions. The construction has two phases, a diagonalization phase in which we take care that $\{0\}^{*}$ is not reduced to $C$ via a many-one reduction that is length increasing on almost all strings, and an encoding phase, in which the strings in $D$ that are also in $K_{\mathrm{NEXP}}^{A}$ are encoded into the oracle in such a way that a nondeterministic exponential time set, here $C$, can compute them on inputs that are exponentially smaller. We use a fast growing but easy to compute function $b(i)$ which is at least double exponential, i.e., $b(i+1)>2^{2^{b(i)}}$.

Phase 1: Diagonalization-In this phase we construct $D$, and part of $A$.

Additional notation: For integers $i$ and $j$, and sets $X$ and $Y$, let $q_{\text {next }}(i, j, X, Y)$ denote the first query of the form $q=\left\langle 0, j^{\prime}, x\right\rangle$, computed by $f^{X}\left(0^{b(i)}\right)$ with $|q|>$ $b(i), x \notin Y, q \notin X$, and $j^{\prime} \leq j$, if any, and $\lambda$ otherwise.

1: Stage 0: $A=\emptyset, D=\emptyset$;

2: Stage $i$ : set $j=1^{i \log b(i)} ; D_{i}=\emptyset ; A_{i}=\emptyset$;

3: while $\left(q=q_{\text {next }}\left(i, j, A \cup A_{i}, D_{i}\right)\right) \neq \lambda$ do

4: $A_{i}=A_{i} \cup\left\{\left\langle 0, c, \pi_{3}(q)\right\rangle \mid 0^{i \log b(i)} \leq c \leq j\right\}$; recall that $\pi_{3}(q)$ is the projection of the third element in $q$.

5: $D_{i}=D_{i} \cup\left\{\pi_{3}(q)\right\} ; j=j-1$;

6: end while

7: if $\left|f_{i}^{A \cup A_{i}}\left(0^{b(i)}\right)\right|>b(i)$ then

8: $\quad D=D \cup D_{i} \cup\left\{f_{i}\left(0^{b(i)}\right)\right\}$;

9: $\quad A=A \cup A_{i} \cup\left\{\left\langle 0, c, f_{i}\left(0^{b(i)}\right)\right\rangle \mid 0^{i \log b(i)} \leq c \leq j\right\}$;

10: end if

Phase 2: Coding-here we make adjustments to the oracle $A$ such that $K^{A}-D$ remains incomplete via length increasing reductions. However, strings in $D$ are reencoded in $A$ such that a NEXP machine can recover these strings on inputs of logarithmic lengths.

1: Stage $x$ :

2: if $x \in K^{A} \cap D$ then

3: $\quad$ Let $c=\max \{i \mid\langle 0, i, x\rangle \in A\}$;

4: $\quad$ Let $k=b(i)^{i}$ where $b(i) \leq|x| \leq b(i)^{i}$;

5: $\quad$ Let $y=\min \left\{z|| z \mid=k^{2} \wedge\langle 1, c, z\rangle \notin Q\left(f_{i}^{A}\left(0^{b}(i)\right)\right) \cup\left\{Q\left(N_{K}^{A}(v)\right) \mid v \leq x\right\}\right.$

6: $\quad A=A \cup\{\langle 1, c, y\rangle\}$;

7: end if 
Interleaving The diagonalization phase and the coding phase can be executed simultaneously, but interleaved. The diagonalization at stage $i$ assumes that the oracle is fixed below length $b(i)$. Therefore the coding of all such strings has to be done before this stage starts. This means that the coding of all $x$ 's in $K^{A} \cap D$ that require changes to the oracle at lesser length have to be encoded before stage $i$. The $x$ 's in $\cup\left\{D_{j} \mid j<i\right\}$ are of length at most $b(i-i)^{i-1}$. To determine whether these $x$ 's are also in $K^{A}$, queries must be made to $A$ at lengths at most $2^{b(i-1)^{i-1}}$ (note that this may be changed by the encoding itself, but not by stages $>i$ of the diagonalization, moreover this encoding stabilizes by the fact that queries of previously encoded strings are avoided). Then these $x$ 's are encoded at length $|x|^{2}$ which is at maximum $\left(b(i-1)^{i-1}\right)^{2}$. However $\left(\forall^{\infty} i\right)\left[b(i-1)^{(i-1)^{2}}<2^{b(i-1)^{i-1}}<b(i)\right]$, since $b(i)$ is double exponential. It follows that diagonalization and coding can be safely interleaved by first doing an entire diagonalization stage, and then coding the strings that entered $D$ during that stage.

Lemma 7 (Room to diagonalize) Whenever strings need to be added to A during the diagonalization phase, there are enough strings not yet determined.

Proof The diagonalization phase during stage $i$ adds strings of length between $b(i)$ and $b(i)^{i}$. The number of these strings is bounded by $b(i)^{i} \times \sum_{j=0}^{b(i)^{i}} j$, which is less than $b(i)^{3 i}$. For all but finitely many $x$ this is less than $2^{b(i)^{i}}$, the number of strings not yet set in the oracle at this length.

Lemma 8 (Room to encode) Whenever strings need to be added to A during the encoding phase, there are enough strings not yet determined.

Proof Whenever a string $x$ needs to be encoded, we search for a string $y$ of length $|x|^{2}$ such that $\langle 1, c, x\rangle$ is not yet in the oracle. Note that strings added by the diagonalization phase are all of the form $\langle 0, u, v\rangle$, so these do not matter. Other strings that could be prohibited from entering the oracle are strings queried in a computation of $M_{K_{\mathrm{NEXP}}}^{A}$, on inputs $y<x$. There are less than $2^{|x|+1}$ of these strings, each giving rise to at most $2^{|y|}$ queries. It follows that this number is bounded by $2^{2|x|+1}$ which is much less than the $2^{|x|^{2}}$ strings available for almost all $x$.

The complete set $C$ is defined as follows.

1: input $\langle i, x\rangle: i \in\{0,1\}$;

2: if $i=0$ and $x \in K^{A}-D$ then accept

3: end if

4: if $(\exists y)\left[|y|=2^{2|x|} \wedge\langle 1, x, y\rangle \in A\right]$ then accept

5: end if

6: reject

\section{Lemma $9 C \in \mathrm{NEXP}^{A}$.}

Proof It follows more or less straightforward from the construction and the way diagonalizing and coding is interleaved that computing whether an input is in $D$ can 
be done in deterministic exponential time. Observe that strings for which nondeterministic exponential time computations must be simulated are all exponentially smaller. $x \in K^{A}$ can be decided in nondeterministic linear exponential time, and an oracle query of length $2^{2|x|}$ can be built in nondeterministic linear exponential time as well.

Lemma $10\{0\}^{*}$ does not reduce to $C$ via a reduction that is almost always length increasing.

Proof Suppose it does, then this length increasing reduction has some index $i$ where $b(i+1)$ is large enough to satisfy all room to diagonalize/code conditions and moreover $M_{i}\left(0^{b(i)}\right)>b(i)$. At stage $i$ the oracle is fixed so that the output $M_{i}\left(0^{b(i)}\right)$ is in $D$ and hence not in $C$, a contradiction.

The final part is the definition of the reduction $F$ from $K^{A}$ to $C$.

1: input $x$

2: Let $k=\max \{i|b(i) \leq| x \mid\}$

3: if $\left\langle 0,0^{i \log b(i)}, x\right\rangle \notin A$ then output $\langle 0, x\rangle$;

4: else

5: $\quad$ Let $\ell=\max \{j \mid\langle 0, j, x\rangle \in A\}$;

6: $\quad$ output $\langle 1, \ell\rangle$;

7: end if

The final lemma for this construction then says that $C$ remains complete.

Lemma $11 K^{A} \leq_{m}^{p} C$.

Proof If $x \notin D$ then $x \in K^{A}$ iff $x \in C$. In this case no string of the form $\langle 0, y, x\rangle$ is in $A$ by construction, so the reduction, which is identity in this case, works. If $x \in D \cap K^{A}$ then for the maximum $c$ such that $\langle 0, c, x\rangle$ is in $A$, there exists a string $y$ of length $|x|^{2}$ with $\langle 1, y, c \in A\rangle$, hence $c \in C$. If $x \in D-K^{A}$ then no such $y$ is in $A$, hence $c \notin C$.

By the following observation, the construction can be adapted to reductions that have $k$ bits of advice by the following observation. In stage $i$ of the construction we diagonalize against many-one reduction $M_{i}$, resulting in the coding of $n^{i}$ strings from $K^{A}$ into strings of length $\log \left(n^{i}\right)=i \log n$. Instead of diagonalizing against just $M_{i}$ the same construction can be used to diagonalize against $2^{k}$ different advices for $M_{i}$ resulting in a coding of $2^{k} n^{i}$ strings from $K^{A}$ into strings of length $\log \left(2^{k} n^{i}\right)=$ $k+i \log n$.

Corollary 12 There exists an oracle witnessing that NEXP has a many one complete set that is not complete under length increasing reductions that use $k$ bits of advice.

The oracle above works for nondeterministic polynomial time as well as nondeterministic exponential time. The only significant change in the constructions above 
is that we pad the counter so that the replacement in $C$ is only polynomially smaller in length than the original element taken from $C$.

Theorem 13 There exists an oracle witnessing that NP has a many one complete set that is not complete with length increasing reductions.

Proof As before, we construct sets $A, C$, and $D$, such that $C=K_{\mathrm{NP}}^{A}-D$ is complete for $\mathrm{NP}^{A}$, but not via length-increasing reductions. Again, the construction has two phases, a diagonalization phase in which we take care that $\{0\}^{*}$ is not reduced to $C$ via a many-one reduction that is length increasing on almost all strings, and an encoding phase, in which the strings in $D$ that are also in $K_{\mathrm{NP}}^{A}$ are encoded into the oracle in such a way that a nondeterministic polynomial time set, here $C$, can compute them on inputs that are polynomially smaller. We use a fast growing but easy to compute function $b(i)$ which is at least double exponential, i.e., $b(i+1)>2^{2^{b(i)}}$.

Phase 1: Diagonalization-In this phase we construct $D$, and part of $A$. Let $q_{\text {next }}(i, j, X, Y)$ be defined as above. Here the major change is the length of our counter $j$, we pad it to $1^{b(i)^{\frac{1}{2 i}}}$ bits.

1: Stage 0: $A=\emptyset, D=\emptyset$;

2: Stage $i$ : set $j=0^{b(i)^{\frac{1}{2 i}}-i \cdot \lg (b(i))} 1^{i \cdot \lg (b(i))} ; D_{i}=\emptyset ; A_{i}=\emptyset$;

3: while $\left(q=q_{\text {next }}\left(i, j, A \cup A_{i}, D_{i}\right)\right) \neq \lambda$ do

4: $A_{i}=A_{i} \cup\left\{\left\langle 0, c, \pi_{3}(q)\right\rangle \mid 0^{b(i)^{\frac{1}{2 i}}} \leq c \leq j\right\}$;

5: $D_{i}=D_{i} \cup\left\{\pi_{3}(q)\right\} ; j=j-1$;

6: end while

7: if $\left|f_{i}^{A \cup A_{i}}\left(0^{b(i)}\right)\right|>b(i)$ then

8: $\quad D=D \cup D_{i} \cup\left\{f_{i}\left(0^{b(i)}\right)\right\}$;

9: $\quad A=A \cup A_{i} \cup\left\{\left\langle 0, c, f_{i}\left(0^{b(i)}\right)\right\rangle \mid 0^{b(i)^{\frac{1}{2 i}}} \leq c \leq j\right\}$;

10: end if

Phase 2: Coding-again adjustments to the oracle $A$ such that $K^{A}-D$ remains incomplete via length increasing reductions, however strings in $D$ are reencoded in $A$ such that an NP machine can recover these strings. Unlike before, these strings are polynomially smaller in length.

1: Stage $x$ :

2: if $x \in K^{A} \cap D$ then

3: $\quad$ Let $c=\max \{i \mid\langle 0, i, x\rangle \in A\}$;

4: $\quad$ Let $k=b(i)^{i}$ where $b(i) \leq|x| \leq b(i)^{i}$;

5: $\quad$ Let $y=\min \left\{z|| z \mid=k^{2} \wedge\langle 1, c, z\rangle \notin Q\left(f_{i}^{A}\left(0^{b}(i)\right)\right) \cup\left\{Q\left(N_{K}^{A}(v)\right) \mid v \leq x\right\}\right.$

6: $\quad A=A \cup\{\langle 1, c, y\rangle\}$;

7: end if

Interleaving As with NEXP the diagonalization phase and the coding phase can be executed simultaneously, but interleaved.

Again because of the size of our function $b$, diagonalization and coding can be safely interleaved by first doing an entire diagonalization stage, and then coding the strings that entered $D$ during that stage. 
Lemma 14 (Room to diagonalize) Whenever strings need to be added to A during the diagonalization phase, there are enough strings not yet determined.

Lemma 15 (Room to encode) Whenever strings need to be added to A during the encoding phase, there are enough strings not yet determined.

The proofs for encoding and diagonalization exactly follow the cases for NEXP. We define a similar complete set $C$, the only difference is the length of our witness.

1: input $\langle i, x\rangle: i \in\{0,1\}$;

2: if $i=0$ and $x \in K^{A}-D$ then accept

3: end if

4: if $(\exists y)[|y|=4|x| \wedge\langle 1, x, y\rangle \in A]$ then accept

5: end if

6: reject

\section{Lemma $16 C \in \mathrm{NP}^{A}$.}

Proof As before, this follows from the construction of the diagonalization and the encoding. We can decide whether an input is in $D$ in polynomial time. $x \in K^{A}$ can be decided in nondeterministic polynomial time, and an oracle query of length $4|x|$ can be built in nondeterministic linear time as well.

Lemma $17\{0\}^{*}$ does not reduce to $C$ via a reduction that is almost always length increasing.

Proof Suppose it does, then this length increasing reduction has some index $i$ where $b(i+1)$ is large enough to satisfy all room to diagonalize/code conditions and moreover $M_{i}\left(0^{b(i)}\right)>b(i)$. At stage $i$ the oracle is fixed so that the output $M_{i}\left(0^{b(i)}\right)$ is in $D$ and hence not in $C$, a contradiction. The final part is the definition of the reduction $F$ from $K^{A}$ to $C$.

1: input $x$

2: Let $k=\max \{i|b(i) \leq| x \mid\}$

3: if $\left\langle 0,0^{i \log b(i)}, x\right\rangle \notin A$ then output $\langle 0, x\rangle$;

4: else

5: $\quad$ Let $\ell=\max \{j \mid\langle 0, j, x\rangle \in A\}$;

6: $\quad$ output $\langle 1, \ell\rangle$;

7: end if

The final lemma for this construction then says that $C$ remains complete.

Lemma $18 K^{A} \leq_{m}^{p} C$.

Proof If $x \notin D$ then $x \in K^{A}$ iff $x \in C$. On the other hand in this case no string of the form $\langle 0, y, x\rangle$ is in $A$ by construction, so the reduction, which is identity in this case works. If $x \in D \cap K^{A}$ then for the maximum $c$ such that $\langle 0, c, x\rangle$ is in $A$ there exists a string $y$ of length $|x|^{2}$ with $\langle 1, y, c \in A\rangle$, hence $c \in C$. If $x \in D-K^{A}$ then no such $y$ is in $A$, hence $c \notin C$. 


\subsection{1-Truth-Table Versus Many-One Reductions}

Sometimes 1-tt reductions can be converted into many-one reductions. Under a rather strong assumption we can prove this theorem for 1-tt reductions that use one bit of advice.

Theorem 19 Let $A$ be $\leq_{1-\mathrm{tt}}^{p}$ complete for NP. If there exists a set $R$ in NP that is $\operatorname{NTIME}\left(2^{c n}\right) \cap \operatorname{co-NTIME}\left(2^{c n}\right)$-bi-immune, then $A$ is also $\leq_{m}^{p / 1}$-complete for NP.

Proof Let $A$ be $\leq_{1-\mathrm{tt}}^{p}$ complete and let

$$
D=\left\{\begin{array}{l}
\langle\phi, 0\rangle: \phi \in \mathrm{SAT} \vee 0^{|\phi|} \in R \\
\langle\phi, 1\rangle: \phi \in \mathrm{SAT} \wedge 0^{|\phi|} \in R
\end{array}\right\} .
$$

Since $R \in \mathrm{NP}, D \in \mathrm{NP}$ and so $D \leq_{1-\mathrm{tt}}^{p} A$. Obviously SAT $\leq_{m}^{p / 1} D$. The single bit of advice needed is whether $0^{|\phi|} \in R$. Let $M$ be the $\leq_{1-\text { tt }}^{p}$ reduction from $D$ to $A$. $M$, on input $\langle\phi, 0\rangle$, produces a string $z$. Then, depending on the program of $M,\langle\phi, 0\rangle \in$ $D \leftrightarrow z \in A$ or $\langle\phi, 0\rangle \in D \leftrightarrow z \notin A$. In the first case we say $M$ is of type $m$ (manyone) on input $\langle\phi, 0\rangle$ and in the second case that $M$ is of type $\bar{m}$ on input $\langle\phi, 0\rangle$. We now claim that the bi-immunity of $R$ implies $M$ can be of type $\bar{m}$ for only finitely many unsatisfiable $\phi$. Or, in other words,

$$
\begin{aligned}
& \left(\exists^{\infty} \phi \notin \mathrm{SAT}\right)[\operatorname{type}(M(\phi, 0))=\bar{m}] \Rightarrow \\
& (\exists B)\left[\|B\|=\infty \wedge B \in \operatorname{NTIME}\left(2^{2 n}\right) \cap \operatorname{co}-\operatorname{NTIME}\left(2^{2 n}\right) \wedge[B \subseteq R \vee B \subseteq \bar{R}]\right] .
\end{aligned}
$$

We first prove this claim. Consider

$$
\begin{aligned}
& C=\left\{0^{n}|\exists \phi \notin \mathrm{SAT},| \phi \mid=n, \operatorname{type}(M(\phi, 0))=\bar{m}\right\}, \\
& \|C\|=\infty \rightarrow[\|C \cap R\|=\infty \vee\|C \cap \bar{R}\|=\infty] .
\end{aligned}
$$

Assume $\|C \cap R\|=\infty$. Set $B=C \cap R$. Now $B \in \operatorname{NTIME}\left(2^{2 n}\right)$ by the following algorithm. On input $0^{n}$ guess $\phi$ with $|\phi|=n$ and $\phi \notin \mathrm{SAT}$; Check that type $(M(\phi, 0))=\bar{m}$ and verify $0^{n} \in R$ using the reduction to $A$. It is also the case that $\bar{B}$ is in $\operatorname{NTIME}\left(2^{2 n}\right)$ by the following algorithm. On input $0^{n}$ check that for every $\phi$ of length $n$ either $\phi \in$ SAT or that type $(M(\phi, 0))=m$, or that there exists a $\phi \notin$ SAT of length $n$ for which type $(M(\phi, 0))=\bar{m}$ but $m(\phi, 0) \in A$ (which means $\left.0^{n} \notin R\right)$.

We conclude that under the assumption of the theorem there can only be finitely many unsatisfiable $\phi$ such that $M(\phi, 0)$ is of type $\bar{m}$. A similar proof shows that there can be only finitely many satisfiable $\phi$ such that $M(\phi, 1)$ is of type $m$. From this we can build our many-one reduction.

The premise of the previous theorem seems rather strong. Yet it is not impossible that NP does have such sets. To provide evidence for this statement we construct precisely such a set in the following theorem.

Theorem 20 For every constant c, there exists an oracle A such that NP has a set that is $\operatorname{NTIME}\left(2^{n^{c}}\right) \cap$ co-NTIME $\left(2^{n^{c}}\right)$-immune. 
Proof We use a construction appearing in [9] in which the oracle is created from an infinite Kolmogorov random string. The language that will have the property stated in the theorem will be the following. For all oracles $X$ define $D_{X}=\{x \mid(\exists y)[|y|=$ $\left.n^{2 c} \wedge x y \in X\right\}$. Let $Y$ be an infinite string that has the property that $(\forall n)\left[K\left(Y_{[1 . . n]}\right)>\right.$ $n^{c}$ ]. We will construct $A$ by stages. $A_{s+1}$ is the oracle defined by the end of stage $s$ and $A_{s+1} \subseteq A_{s}$ for all $s$. The oracle $A$ may have $2^{n}$ strings of length $n+n^{2 c}$. We now describe the initial oracle $A_{0}$.

To encode a string $x$ into $D$, we need a sequence of $|x|^{2 c}$ bits. For this encoding we use substrings of $Y$. Up to $|x|$ we have used bits of $Y$ for $\sum_{i<|x|} 2^{i} \leq 2^{|x|}$ strings, so if we let the substrings of $Y$ that encode strings of length $|x|$ start at the $2^{|x|} \times|x|^{2 c}$ th bit of $Y$, there will never be a conflict (two strings in $A$ taking their encoding from the same substring of $Y$, note that strings smaller than $x$ require less bits for encoding). Moreover, we have sequence from the $2^{|x|} \times|x|^{2 c}$ th bit up until the $2^{|x|+1} \times|x|^{2 c}$ th bit of $2^{|x|}$ substrings of length $|x|^{2 c}$ that can be used to encode strings of length $|x|$. The encoding of strings of length $|x|+1$ then starts at the $2^{|x|+1} \times|x+1|^{2 c}$ th bit of $Y$, which is quite a bit further along. For $A_{0}$ up to the $i$ th string of length $m$ we use a substring of $Y$, say $Y_{A_{0}}^{m, i}$ of length $2^{m} m^{2 c}+i m^{2 c}$ bits. The incompressibility of $Y$ implies that for some constant $d$ and all $m$ and $i$ it holds that $K\left(Y_{A_{0}}^{m, i}\right) \geq 2^{m} m^{2 c}+$ $i m^{2 c}-d$. This is the property we will use to prove correctness of our construction. This ends the description of the initial oracle $A_{0}$. We shall next describe a stage construction of the oracle that has the desired properties.

At each stage $s$ we will decide the membership of the string $x_{s}$ in $D^{A}$, where $x_{s}$ is the $s$ th string in the lexicographical ordering of $\Sigma^{*}$. Deciding membership of $x_{s}$ is deciding whether to remove $x_{s} y$ from $A$ where $x_{s} y$ is the only string that is an extension of $x_{s}$ currently in $A$. The Kolmogorov property that makes the entire construction work is that a (nondeterministic) machine that rejects some string $x_{s}$ with oracle $A_{s}$ must also reject $x_{s}$ with oracle $A$ or else it will allow us-via a description of the position of a query on the least accepting path-to describe some initial segment of $Y$ using significantly less bits than the length of this segment, which then violates the aforementioned Kolmogorov property.

Let $\left\{M_{i}\right\}_{i}$ be an enumeration of all nondeterministic Turing machines, where $2^{n^{c}}$ is the time bound on inputs of length $n$ for all machines in the enumeration. The construction maintains two sets of requirements. First, a set $U_{s}$ of yet unsatisfied requirements to which occasionally a new element is added and from which satisfied requirements are removed. Second, a set $V_{s}$ in which satisfied requirements are kept. Sometimes a satisfied requirement will be moved from $V_{s}$ to $U_{s}$ at which time it will become unsatisfied again. Every requirement corresponds to a language in NTIME $\left(2^{n^{c}}\right) \cap \operatorname{co}-\operatorname{NTIME}\left(2^{n^{c}}\right)$, represented by nondeterministic $2^{n^{c}}$-time bounded machines $M_{i}$ and $M_{j}$. Even requirements $R_{2\langle i, j\rangle}$ will represent the need to establish a nonempty intersection of $L\left(M_{i}\right) \cap \overline{L\left(M_{j}\right)}$ with $D$, whereas odd requirements $R_{2\langle i, j\rangle+1}$ will represent the need to establish a nonempty intersection of $L\left(M_{i}\right) \cap \overline{L\left(M_{j}\right)}$ with $\bar{D}$. 
We will prove that the construction of $A$ meets the following requirements for all $\langle i, j\rangle$.

1. $R_{2\langle i, j\rangle}:\left\|L\left(M_{i}^{A}\right)\right\|=\infty \Longrightarrow\left[\left[L\left(M_{i}^{A}\right) \neq \overline{L\left(M_{j}^{A}\right)}\right] \vee\left[L\left(M_{i}^{A}\right) \cap D_{A} \neq \emptyset\right]\right]$.

2. $R_{2\langle i, j\rangle+1}:\left\|L\left(M_{i}^{A}\right)\right\|=\infty \Longrightarrow\left[\left[L\left(M_{i}^{A}\right) \neq \overline{L\left(M_{j}^{A}\right)}\right] \vee\left[L\left(M_{i}^{A}\right) \cap \overline{D_{A}} \neq \emptyset\right]\right]$.

There are two ways to fulfill these requirements. Either maintain a difference between $L\left(M_{i}\right)$ and $\overline{L\left(M_{j}\right)}$, i.e., the pair $M_{i}, M_{j}$ does not represent a language in $\operatorname{NTIME}\left(2^{n^{c}}\right) \cap \operatorname{co}-\operatorname{NTIME}\left(2^{n^{c}}\right)$, or maintain a string in the intersection of $L\left(M_{i}\right)$ and $D$, respectively $\bar{D}$.

A requirement $R_{e}$ with $e=2\langle i, j\rangle$, or $e=2\langle i, j\rangle+1$ is active at stage $s$ if $x_{s} \in$ $L\left(M_{i}^{A_{s}}\right)$. Now the construction can be described as follows.

1: stage $s$ :

2: if no requirement is active then

3: $\quad A_{s+1}=A_{s}$;

4: $\quad V_{s+1}=V_{s} ; U_{s+1}=U_{s} ;$

5: else

6: $\quad$ let $e$ be the least active requirement in $U_{s}$;

7: $\quad$ if $e$ is even then

8: $\quad U_{s+1}=U_{s}-\{e\}$;

9:

10:

11:

18:

19: end if

$V_{s+1}=V_{s} \cup\left\{\left\langle e, x_{s}\right\rangle\right\} ; A_{s+1}=A_{s} ;$

else

$$
\text { if }\left\{\langle i, x\rangle \in V_{s} \mid(i<e) \wedge\left(2^{|x|^{c}} \geq\left|x_{s}\right|\right)\right\}=\emptyset \text { then }
$$

$V_{s+1}=V_{s}-\left\{\langle i, x\rangle \in V_{s} \mid i>e\right\}$

$U_{s+1}=U_{s} \cup\left\{i \mid(i>e) \wedge(\exists x)\left[\langle i, x\rangle \in V_{s}\right]\right\}-\{e\} ;$

$A_{s+1}=A_{s}-\left\{\left\langle x_{s}, y_{s}\right\rangle\right\}$

else

$$
A_{s+1}=A_{s} ; V_{s+1}=V_{s} ; U_{s+1}=U_{s} ;
$$

end if

\section{end if}

20: if $s=2^{k}$ then $U_{s+1}=U_{s+1} \cup\{k\}$.

21: end if

We will now prove correctness of our construction in a series of lemmas. The key lemma in the proof is the following.

Lemma $21\left(\forall^{\infty} s\right)(\forall e<\log s)\left[x_{s} \notin L\left(M_{e}^{A_{s}}\right) \Longrightarrow x_{s} \notin L\left(M_{e}^{A}\right)\right]$.

Proof Suppose not. Let $e$ and $s$ be such that $e<\log s$ and $x_{s} \in L\left(M_{e}^{A}\right)-L\left(M_{e}^{A_{s}}\right)$. Let $s^{\prime}$ be minimal such that $s^{\prime} \geq s$ and $x_{s} \in L\left(M_{e}^{A_{s^{\prime}+1}}\right)-L\left(M_{e}^{A_{s^{\prime}}}\right)$. By construction $A_{s^{\prime}}-$ $A_{s^{\prime}+1}=\left\{x_{s^{\prime}} y_{s^{\prime}}\right\}$. The string $x_{s^{\prime}} y_{s^{\prime}}$ must be queried in any accepting computation of $M_{e}^{A_{s^{\prime}+1}}$ on input $x_{s}$. Otherwise $M_{e}^{A_{s^{\prime}}}$ would also have an accepting computation on input $x_{s}$ contradicting the assumption. On input $x_{s}$, machine $M_{e}$ can only query strings of length less than or equal to $2^{\left|x_{s}\right|^{c}}$. Moreover $\left\|\left\{y \mid y \in A_{0}-A_{s^{\prime}+1}\right\}\right\|<\log s^{\prime}$ since for every such $y$ there is an odd index in $U_{t}-\left(U_{t+1} \cup V_{t+1}\right)$ for some $t<s^{\prime}$ and 
there are no more than $\log s^{\prime}$ indices in $\cup\left\{U_{t} \mid t \leq s^{\prime}\right\}$. Let $n$ be such that $n^{2 c}>\left|x_{s^{\prime}}\right|^{2 c}$. We will show how to construct the first $2^{n+1} n^{2 c}$ bits of $Y$ using significantly less bits and hence arrive at a contradiction. Suppose that we have $2^{n+1} n^{2 c}-\left|x_{s^{\prime}}\right|^{2 c}$ bits of $Y$ that describe the initial segment of $Y$ used to encode $A_{0}$ for all substrings of $Y$ up to and including strings of length $n+n^{2 c}$, except $y_{s^{\prime}}$ (for strings of length $n+n^{2 c}$ there is a corresponding substring of length $n^{2 c}$ in $Y$ as explained above. So $y_{s^{\prime}}$ in $Y, x_{s^{\prime}} y_{s^{\prime}}$ in $\left.A_{0}\right)$. Furthermore suppose that we have a list of at most $\log s^{\prime}$ strings $x_{i_{1}}, \ldots, x_{i_{k}}$ that says which $x_{i_{j}} y_{i_{j}}$ are in $A_{0}-A_{s}^{\prime}$. Note that no strings greater than $x_{s^{\prime}} y_{s^{\prime}}$ are in $A_{0}-A_{s}^{\prime}$. Finally let $q<2^{\left|x_{s}\right|^{c}}$ be the index of the query $x_{s^{\prime}} y_{s^{\prime}}$ in the leftmost accepting computation of $M_{e}^{A_{s^{\prime}+1}}\left(x_{s}\right)$. Note that $q$ requires at most $|x|^{c}$ bits. Now we can construct the first $2^{n+1} n^{2 c}$ bits of $Y$ from $2^{n+1} n^{2 c}-\left|x_{s^{\prime}}\right|^{2 c}+\log s^{\prime} \times$ $\left|x_{s^{\prime}}\right|+\left|x_{s}^{\prime}\right|+\left|x_{s}\right|^{c}+O(1)$ bits of information. We arrive at a contradiction for all but finitely many $s$. The difference between $\left|x_{s}^{\prime}\right|^{2 c}$ and $\log s^{\prime} \times\left|x_{s^{\prime}}\right|+\left|x_{s}^{\prime}\right|+\left|x_{s}\right|^{c}+O$ (1) will outgrow any constant, and so the complexity assumption on $Y$ will be violated.

Lemma $22(\forall e)\left[\left\|\left\{s \mid(\exists x)\left[\langle e, x\rangle \in V_{s+1}-V_{s}\right]\right\}\right\|<\infty\right]$.

Proof Whenever $\langle e, x\rangle$ is in $V_{s+1}-V_{s}$ there is a smaller index that is moved from $U_{s+1}$ either to oblivion or to $V_{s+1}$. This means that 0 can enter $V_{s}$ for only one $s$ and there is no $t$ such that $\langle 0, x\rangle$ is in $V_{t}-V_{t+1}$. By induction for $i<e$, let $\# i$ be the number of times that index $i$ is in $V_{t+1}-V_{t}$ for some $t$ and $x$. Then it is clear that $\# e \leq \sum_{i=0}^{e-1}(1+\# i)$ which is finite for all $e$.

Corollary $23(\forall e)\left[\left(\exists^{\infty} s\right)\left[e \in U_{s}\right] \Longrightarrow\left(\forall^{\infty} s\right)\left[e \in U_{s}\right]\right]$.

Lemma 24 If $\left\|L\left(M_{e}^{A}\right)\right\|=\infty$ then $L\left(M_{e}^{A}\right) \cap D_{A} \neq \emptyset$.

Proof Let $e$ be such that $\left\|L\left(M_{e}^{A}\right)\right\|=\infty$ and $L\left(M_{e}^{A}\right) \cap D_{A}=\emptyset$. If $2 e$ in $V_{s+1}$ then $L\left(M_{e}^{A_{s+1}}\right) \cap D_{A^{s+1}} \neq \emptyset$. Since $L\left(M_{e}^{A}\right) \cap D_{A}=\emptyset$ there are infinitely many $s$ such that $2 e \notin V_{s}$. It follows from Corollary 23 that $2 e \in U_{s}$ for almost all $s$. Consequently, there are infinitely many $s$ such that $x_{s} \in L\left(M_{e}^{A}\right)$ where $2 e \in U_{s}$ and $e<\log s$. At stage $s$ there are two possibilities.

1. $x_{s} \in L\left(M_{e}^{A_{s}}\right)$.

2. $x_{s} \notin L\left(M_{e}^{A_{s}}\right)$.

In case 1 , since $x_{s} \notin D_{A}$ there is some $e^{\prime}<e$ such that $2 e^{\prime}+1 \in U_{s+1}-U_{s}$. Hence case 1 can appear only finitely often. Case 2 must appear infinitely often. Consider a stage where case 2 appears. Then we have $e<\log s$ and $x_{s} \in L\left(M_{e}^{A}\right)-L\left(M_{e}^{A_{s}}\right)$, so it follows from Lemma 21 that this can also appear only finitely often, a contradiction.

Lemma 25 If $L\left(M_{i}^{A}\right)=\overline{L\left(M_{j}^{A}\right)}$ and $\left\|L\left(M_{i}^{A}\right)\right\|=\infty$ then $L\left(M_{i}^{A}\right) \cap \overline{D_{A}} \neq \emptyset$.

Proof We will first argue that for almost all $i$ and $j$, if $2\langle i, j\rangle+1$ is in $U_{s}-\left(U_{s+1} \cup\right.$ $\left.V_{s+1}\right)$ then $L\left(M_{i}^{A}\right) \cap \overline{D_{A}} \neq \varnothing$. Note that a particular language in $\operatorname{NTIME}\left(2^{n^{c}}\right) \cap$ 
$\operatorname{co-NTIME}\left(2^{n^{c}}\right)$ is represented by infinitely many pairs $\langle i, j\rangle$. Suppose that for some (large) $s$ the index $2\langle i, j\rangle+1$ is in $U_{s}-\left(U_{s+1} \cup V_{s+1}\right)$. This means that $x_{s} \in \overline{D_{A}}$ and $x_{s} \notin L\left(M_{j}^{A_{s}}\right)$. It follows from Lemma 21 that $x_{s} \notin L\left(M_{j}^{A}\right)$ and hence from the assumption $L\left(M_{i}^{A}\right)=\overline{L\left(M_{j}^{A}\right)}$ that $x_{s} \in L\left(M_{i}^{A}\right)$. If $\left(\forall^{\infty} s\right)(\exists x)\left[\langle e, x\rangle \in V_{s}\right]$ then let $t$ be maximal so that $e \notin V_{t}$. Since $A_{t}=A_{t+1}$, it is then the case that $x_{t} \in L\left(M_{i}^{A_{t+1}}\right) \cap L\left(M_{j}^{A_{t+1}}\right)$ and moreover since $\left\langle e, x_{t}\right\rangle \in V_{u}$ for all $u \geq t+1$, subsequent changes to the oracle are made only at lengths greater than $2^{\left|x_{t}\right|^{c}}$ hence $x_{t} \in$ $L\left(M_{i}^{A}\right) \cap L\left(M_{j}^{A}\right)$. In this case there is nothing to prove. Finally, let $e=2\langle i, j\rangle+1$ be such that $L\left(M_{i}^{A}\right)=\overline{L\left(M_{j}^{A}\right)}$ and $\left\|L\left(M_{i}^{A}\right)\right\|=\infty$ and $L\left(M_{i}^{A}\right) \subseteq D_{A}$ and moreover $\left(\forall^{\infty} s\right)\left[e \in U_{s}\right]$. By Corollary 23 also $\left(\forall^{\infty} s\right)\left(\forall e^{\prime} \leq e\right)\left[e^{\prime} \in U_{s} \Longrightarrow e^{\prime} \in U_{s+1}\right]$, i.e., no requirement of higher priority will be satisfied at stage $s$. Consider only two stages where $x_{s} \in L\left(M_{i}^{A}\right)$ and $\left(\forall e^{\prime} \leq e\right)\left[e^{\prime} \in U_{s} \Longrightarrow e^{\prime} \in U_{s+1}\right]$. At any of these stages there are two possibilities.

1. $x_{s} \in L\left(M_{i}^{A_{s}}\right)$.

2. $x_{s} \notin L\left(M_{i}^{A_{s}}\right)$.

In the first case $2\langle i, j\rangle+1 \notin U_{s+1}$ contradicting the assumption and in the second case $x_{s} \in L\left(M_{i}^{A}\right)-L\left(M_{i}^{A_{s}}\right)$ so this case can occur only finitely often according to Lemma 21.

The following lemma concludes the proof.

Lemma $26\left\|D_{A}\right\|=\infty$ and $\left\|\overline{D_{A}}\right\|=\infty$.

Proof This is immediate from the fact that both sets have nonempty intersections with languages in $\operatorname{NTIME}\left(2^{n^{c}}\right) \cap \operatorname{co}-\operatorname{NTIME}\left(2^{n^{c}}\right)$ at infinitely many different points $x_{s}$.

\section{When Does Advice Help?}

As we stated in the introduction, reductions with advice seem to inhabit a higher level of complexity than do uniform reductions. In the next theorem we show a simple diagonalization that demonstrates incomparability of uniform 2-tt reductions and many-one reductions with advice.

Theorem 27 There exists a set $A \in \mathrm{EXP}$ that is $\leq_{2-\mathrm{tt}}^{p}$-hard for EXP but not $\leq_{m}^{p / 1}$ hard for EXP, and there exists a set $A^{\prime} \in \mathrm{EXP}$ that is $\leq_{m}^{p / 1}$-hard for EXP but not $\leq_{2-\mathrm{tt}^{p}}^{p}$ hard for EXP.

Proof First we show that there is a set $A \in \mathrm{EXP}$ that is $\leq_{2-\mathrm{tt}}^{p}$-hard for EXP but not $\leq_{m}^{p / 1}$-hard for EXP. We start with an enumeration $\left\{M_{i}\right\}_{i}$ of all polynomial time transducers that take one bit of advice and look at the outcome of the computations on 
input $0^{n}$ for $n$ spaced exponentially far apart. Our exponential-time computable set $D$ that we use for diagonalization is a subset of $\{0\}^{*} \cup\{1\}^{*}$, where strings of this type appear with exponential gaps between them. Our complete set $A$ is a subset of $\{\langle b, x\rangle \mid b \in\{0,1\}\}$, and the 2 -tt reduction from $K_{\mathrm{EXP}}$ to $A$ on input $x$ computes $\langle 0, x\rangle \in A \oplus\langle 1, x\rangle \in A$ and accepts if the outcome is 1 . On suitable inputs $0^{n}$ we diagonalize against $M_{i}$. If $M_{i}$ accepts or rejects without querying, or queries a part of $A$ that has already been set, i.e., exponentially smaller than the input, we diagonalize by letting $M_{i}\left(0^{n}\right)$ compute the wrong answer. If the queries are not of the form $\langle b, x\rangle$ then we do the same, so for the rest of the proof we assume that $M_{i}$ on input $0^{n}$ computes a query that has not yet been set of the form $\langle b, x\rangle$, given advice 0 or 1 . There are several possibilities:

1. $M_{i}$ computes $\left\langle b_{1}, x\right\rangle$ with advice 0 and $\left\langle b_{2}, y\right\rangle$ with advice 1 where $x \neq y$. Then we set $0^{n} \in D,\left\langle b_{1}, x\right\rangle \notin A$, and $\left\langle b_{2}, y\right\rangle \notin A$, furthermore, set $\left\langle 1-b_{1}, x\right\rangle$ in $A$ if $x \in K$, and set $\left\langle 1-b_{2}, y\right\rangle$ in $A$ if $y \in K$.

2. $M_{i}$ computes $\langle b, x\rangle$ both with advice 0 and 1 . Then we set $0^{n} \in D$ and set $\langle 1-$ $b, x\rangle$ to reflect $x \in K$ correctly.

3. $M_{i}$ computes $\langle b, x\rangle$ with advice 0 and $\langle 1-b, x\rangle$ with advice 1 . Now we set $0^{n} \in D$ and compute $M_{i}\left(1^{n}\right)$ with advice 0 and 1 . The case of small query strings is treated in the same way, and the cases above are treated in the same way. If we cannot already diagonalize on $1^{n}$ alone then it must be the case that $M_{i}\left(1^{n}\right)$ computes $\left\langle b_{y}, y\right\rangle$ and $\left\langle 1-b_{y}, y\right\rangle$. There are three cases.

(a) $x=y$ and $b=b_{y}$. In this case we set $0^{n} \in D$ and $1^{n} \notin D$. Set $\langle b, x\rangle$ and $\langle 1-b, x\rangle$ consistent with $x \in K$.

(b) $b=1-b_{y}$. In this case we set $0^{n} \in D, 1^{n} \in D$, and $M^{0}\left(0^{n}\right)=M^{1}\left(1^{n}\right) \notin A$.

(c) $x \neq y$. In this case we set $0^{n} \in D$ and $1^{n} \in D$ and let $M^{0}\left(0^{n}\right) \notin A$ and $M^{1}\left(1^{n}\right) \notin A$.

In the reverse case we have to deal with an enumeration of polynomial time truthtables. We use the same complete set, but use the advice to either produce $\langle 0, x\rangle$ or $\langle 1, x\rangle$ on input $x$. There are again some cases to consider, but in this case we only need a subset of $\{0\}^{*}$ to diagonalize.

Yet reductions with advice and the induced completeness notions show familiar properties. Sets complete for EXP are complete under length-increasing, one-one reductions and are dense.

Theorem 28 If $A$ is complete for EXP under $\leq_{m}^{p / 1}$-reductions, then $A$ is complete for EXP under length-increasing $\leq_{m}^{p / 1}$-reductions as well.

Proof Let $\left\{M_{i}\right\}_{i}$ be an enumeration of polynomial time many-one reductions that use one bit of advice. Let $D$ be defined as follows. For $b \in\{0,1\}$ if $\left|M_{i}^{b}(\langle b, i, x\rangle)\right| \leq|x|$ then let $\langle b, i, x\rangle \in D$ iff $x \notin A$. Else let $\langle b, i, x\rangle \in D$ iff $x \in K$. Since $A$ is complete for EXP, one of the reductions, say $j$ must compute the reduction from $D$ to $A$. For this reduction, it is never the case that the $\left|M_{j}(\langle b, i, x\rangle)\right| \leq|x|$ when $b$ is the correct advice for $|\langle b, i, x\rangle|$. Hence the reduction $f(x)=M_{j}(\langle b, i, x\rangle)$ where $b$ is the correct advice for $|\langle b, i, x\rangle|$ is a $\leq_{m}^{p / 1}$ length-increasing reduction from $K$ to $A$. 
Theorem 29 Let A be complete for EXP under $\leq_{m}^{p / 1}$ reductions, then $A$ is dense.

Proof We construct a set $W$ in EXP as follows. For $b \in\{0,1\}$ and $x \in\{0,1\}^{*}$. If there is an $x^{\prime}<x$ such that $M_{i}^{b}(\langle b, i, x\rangle)=M_{i}^{b}\left(\left\langle b, i, x^{\prime}\right\rangle\right)$, then $\langle b, i, x\rangle \in W \leftrightarrow$ $\left\langle b, i, x^{\prime}\right\rangle \notin W$. Otherwise $\langle b, i, x\rangle \in W$. The set $W$ is exponential time computable and dense (it has at least one of $\langle 0, i, x\rangle,\langle 1, i, x\rangle$ for every $i$ and $x$ ). The reduction from $W$ to $A$ cannot have collisions for the correct advice, hence $A$ must also be dense.

Theorem 30 Let A be complete in EXP under $\leq_{m}^{p / 1}$-reductions, then A is also complete under $\leq_{m, 1-1, l i}^{p / 1}$-reductions.

Proof Let $K$ be the canonical EXP-complete set. Let $K_{0}=\left\{\left\langle 0^{n}, x\right\rangle \mid n \in N, x \in K\right\}$. Clearly, $K_{0}$ is $\leq_{m, 1-1, l i}^{p}$-complete in EXP. We show a $\leq_{m, 1-1, l i}^{p / 1}$-reduction from $K_{0}$ to $A$.

Let $\left\{M_{i}\right\}_{i}$ be an enumeration of polynomial time many-one reductions that take one bit advice. Let $M_{i}^{b}(x)$ denote the output of machine $i$ on input $x$ using advice $b$. First we use the constructions of Theorems 28 and 29 to find a reduction $M_{k}$ from $K_{0}$ to $A$ that is both length-increasing, and 1-1 on strings of the same length. That is, if $b$ is the correct advice for $|x|$ then $(\forall x)\left[\left|M_{k}^{b}(x)\right|>|x|\right]$ and $(\forall x, y)[|x|=|y| \rightarrow$ $\left.M_{k}^{b}(x) \neq M_{k}^{b}(y)\right]$.

Without loss of generality, $M_{k}$ runs in time $n^{k}$ for almost all inputs, and hence for almost all $x, y$ and $b$ if $|y|>|x|^{k}$ then $M_{k}^{b}(x) \neq M_{k}^{b}(y)$. Now let $y_{0}(x)=\min \{y \mid$ $\left.(\exists \ell)\left[y=0^{2^{(k+1) \ell}-|x|} x\right]\right\}$ and define $f^{b}(x)=M_{k}^{b}\left(y_{0}(x)\right)$.

Note that $f$ is polynomial time computable. For every $x$ the length of $y_{0}(x)$ is at most $2^{k+1}|x|$, so that the computation of $M_{k}$ on $y_{0}(x)$ takes at most $2^{k^{2}}|x|^{k}$ time, which is polynomial in $|x|$. Next, if $x \neq x^{\prime}$ then either $\left|y_{0}(x)\right|=\left|y_{0}\left(x^{\prime}\right)\right|$ in which case $f^{b}(x) \neq f^{b}\left(x^{\prime}\right)$, for $b$ the correct advice for length $\left|y_{0}(x)\right|$ by properties of $M_{k}$, or $\left|y_{0}(x)\right| \neq\left|y_{0}\left(x^{\prime}\right)\right|$ but then, without loss of generality, assume $y_{0}\left(x^{\prime}\right)$ to be the longer string $\left|y_{0}\left(x^{\prime}\right)\right| \geq\left|y_{0}(x)\right|^{k}$, then $f^{b}(x) \neq f^{b^{\prime}}\left(x^{\prime}\right)$ for $b$ the correct advice for length $\left|y_{0}(x)\right|$ and $b^{\prime}$ the correct advice for length $\left|y_{0}\left(x^{\prime}\right)\right|$ by the fact that $M_{k}$ is length increasing and $n^{k}$-time computable. In both cases $f$ is 1-1. The advice given to $f$ on input $x$ is the advice belonging to $M_{k}$ on input $y_{0}(x)$, which depends only on $|x|$.

However, unlike the uniform case, EXP has a $\leq_{m}^{p / 1}$-complete set that is P-biimmune.

Theorem 31 There exists $a \leq_{m}^{p / 1}$-complete set in EXP that is $\mathrm{P}$-bi-immune.

We construct a set $A$ complete under $\leq_{m}^{p / 1}$-reductions that is P-bi-immune by creating $A$ in such a way that every infinite polynomial time language has an element in $A$ and in $\bar{A}$. We construct such an $A$ length by length in stages. At every stage, $n$, we consider the polynomial time machines with a smaller index than $n$, and we do so by picking the minimally indexed machine that has not accepted an element in $A$ 
or $\bar{A}$. Specifically, define $R_{i}^{\bar{A}}$ to be the requirement that $M_{i}$ accepts some element in $\bar{A}$ and $R_{i}^{A}$ to be the requirement that $M_{i}$ accepts some element of $A$. The requirements are given priority as $R_{0}^{\bar{A}}>R_{0}^{A}>R_{1}^{\bar{A}}>R_{1}^{A} \ldots$ We will code $K$ the standard manyone complete set for EXP into A length by length. We do this encoding by adding in strings of the form $\langle b, x\rangle$. Here we will use the bit $b$ to diagonalize against the polynomial time machines, we keep $A \leq_{m}^{p / 1}$-complete by using the same bit $b$ at each length, $n$.

Proof Let $\left\{M_{i}\right\}_{i}$ be an enumeration of polynomial time computable sets. Let $K$ be the standard $\leq_{m}^{p}$ EXP complete set. We construct $A$ in stages at each stage $n$ we create set $A_{n}$ and $A=\bigcup_{i \in \mathbb{N}} A_{i}$

\section{Stage n:}

1. Find the minimally indexed machine $M_{i}$ with $i<n$, with either $\| L\left(M_{i}\right) \cap$ $A_{n-1}\|=\| L\left(M_{i}\right) \|$ or $\left\|L\left(M_{i}\right) \cap \overline{A_{n-1}}\right\|=\left\|L\left(M_{i}\right)\right\|$ that accepts some $\langle b, x\rangle$ with $|\langle b, x\rangle|=n$, where $b \in\{0,1\}, x \in\{0,1\}^{*}$. If such a machine and $\langle b, x\rangle$ exist then do one of the following:

(a) If $\left\|L\left(M_{i}\right) \cap A_{n}\right\|=\left\|L\left(M_{i}\right)\right\|$ leave $\langle b, x\rangle$ and all $\langle b, y\rangle$, with $|\langle b, y\rangle|=n$ out of $A_{n}$. Then $\forall\langle 1-b, y\rangle,|\langle 1-b, y\rangle|=n$, put $\langle 1-b, y\rangle \in A_{n} \leftrightarrow y \in K$. (We have fulfilled requirement $R_{i}^{\bar{A}}$.)

(b) Else $\left\|L\left(M_{i}\right) \cap \overline{A_{n}}\right\|=\left\|L\left(M_{i}\right)\right\|$ and we put $\langle b, x\rangle$ in $A$. Then $\forall\langle b, y\rangle$, $|\langle b, y\rangle|=n$, put $\langle b, y\rangle \in A_{n} \leftrightarrow y \in K$. (We have fulfilled requirement $R_{i}^{A}$.)

2. If no machine $M_{i}$ with $i<n$ accepts a $\langle b, x\rangle$ with $|\langle b, x\rangle|=n$, then we need only keep $A$ complete, $\forall\langle 0, y\rangle,|\langle 0, y\rangle|=n$, put $\langle 0, y\rangle \in A_{n} \leftrightarrow y \in K$.

We claim that for every $M_{i}$ with $\left\|L\left(M_{i}\right)\right\|=\infty, L\left(M_{i}\right) \cap A \neq \emptyset$ and $L\left(M_{i}\right) \cap$ $\bar{A} \neq \emptyset$. First we show $L\left(M_{i}\right) \cap \bar{A} \neq \emptyset$. Assume not, namely, $\left\|L\left(M_{i}\right)\right\|=\infty$, $L\left(M_{i}\right) \subseteq A$. Notice that at some stage $n, M_{i}$ will be the minimally indexed machine that accepts some $\langle b, x\rangle$. Since $\left\|L\left(M_{i}\right)\right\|=\infty$ and $L\left(M_{i}\right) \subseteq A$ it will eventually accept some $\langle b, x\rangle$. We leave that pair out and we have an element in $\bar{A}$ and a contradiction.

The same argument works for the complement. Assume that for some machine $M_{i}$, $\left\|L\left(M_{i}\right)\right\|=\infty$ and, $L\left(M_{i}\right) \subseteq \bar{A}$. Again at some stage $n, M_{i}$ will be the minimally indexed machine that accepts some $\langle b, x\rangle$. This time we put $\langle b, x\rangle$ in $A$ and obtain our contradiction.

Note that for a machine which accepts only a finite number strings or strings of a different form we are unable to fulfill the corresponding requirement, this does not influence our result as this machine cannot accept an infinite subset in $A$ or $\bar{A}$.

At the cost of more queries, in particular at the cost of adaptive queries, there does exist a relation between completeness under reductions that use advice and uniform reductions as the following theorem shows.

Theorem 32 Every set $A$ that is $\leq_{m}^{p / 1}$ complete in EXP is also $\leq_{T}^{p}$-complete in EXP. 
Proof Let $A$ be some $\leq_{m}^{p / 1}$ complete set, let $K$ be the standard many-one complete set. We will create a Turing reduction from $K$ to $A$ by using a few intermediate sets in EXP

These sets will be based on the tableau representation of the exponential time machine computing $x \in K$. Let the EXP machine computing $K$ be $M_{K}$. We assume the running time of $M_{K}$ is $2^{p(n)}$ for some polynomial $p$. Consider an exponential size rectangle that is an encoding of the computation $x \in K$. Where the $i$ th row denotes the tape contents during the $i$ th step of the computation. The columns of the rectangle refer to the contents of a particular tape cell throughout each step of the computation. We call this rectangle the tableau of the computation $x \in K$. Say, a bit in this computation has position $i, j$, where $1 \leq i, j \leq 2^{p(n)}$, and assume that the final bit in this computation is 0 or 1 representing reject and accept respectively. Moreover, by padding $i, j$, where $1 \leq i, j \leq 2^{p(n)}$, we can ensure that for every $x,\langle x, i, j\rangle$ is of the same length for every $\langle i, j\rangle$. (Namely, $i, j$ will be binary strings between $0^{p(n)} 1$ and $\left.10^{p(n)}.\right)$

Now consider the language $B$, the set of $\langle i, j, x\rangle$ with the following conditions:

1. $\langle i, j\rangle$ are each padded to length $p(|x|)+1$.

2. $\langle i, j\rangle$ bit in the tableau for $M_{K}(x)$ is 1 .

First notice that $B \in \mathrm{EXP}$, and as $A$ is $\leq_{m}^{p / 1}$-complete, there is a polynomial time machine, $M_{B}^{b}$, where for correct the advice $b,\langle i, j, x\rangle \in B \leftrightarrow M_{B}^{b}(\langle i, j, x\rangle) \in A$. Also notice there is a many-one reduction from $K$ to $B$, i.e. $x \in K \leftrightarrow$ $\left\langle 10^{p(|x|)}, 10^{p(|x|)}, x\right\rangle \in B$. Now for all $i, j, i^{\prime}, j^{\prime}$ between $0^{p(n)} 1$ and $10^{p(n)}$, $\langle i, j, x\rangle \in B$ corresponds to the $\langle i, j\rangle$ bit of $M_{K}(x)$ and $|\langle i, j, x\rangle|=\left|\left\langle i^{\prime}, j^{\prime}, x\right\rangle\right|$. This means that the same advice bit is used by $M_{B}^{b}$ on all $\langle i, j, x\rangle$ to compute the bits of the exponential computation by $M_{K}$. Since we would like to remove the advice consider the computation of $M_{B}^{0}$ and $M_{B}^{1}$ over all $\langle i, j, x\rangle$ with $i, j$ between $0^{p(n)} 1$ and $10^{p(n)}$. Let the bits computed by $M_{B}^{0}$ be the 0 -tableau and the bits by $M_{B}^{1}$ be the 1 -tableau. Note that if the final bits in the 0-tableau and the 1-tableau are the same, then we know the answer to $x \in K$.

If the final bits are not the same, then there must be an inconsistency for exactly one of the two tableaux (the one computed with the incorrect advice). Now we will show how to recover the correct advice using the inconsistency in the tableau. Consider the set $B^{\prime}$ defined as:

$$
\begin{aligned}
B^{\prime}= & \{\langle b, i, j, x\rangle \\
& \left.\mid\left[\langle i, j, x\rangle \in B \wedge M_{B}^{b}(\langle i, j, x\rangle) \in A\right] \vee\left[\langle i, j, x\rangle \notin B \wedge M_{B}^{b}(\langle i, j, x\rangle) \notin A\right]\right\} .
\end{aligned}
$$

For the correct advice, $b$, to the reduction $M_{B},\langle b, i, j, x\rangle \in B^{\prime}$ for all $i, j$ between $0^{p(n)} 1$ and $10^{p(n)}$. For the incorrect advice, $b^{\prime}$, there is some $\langle i, j\rangle$ with $\langle b, i, j, x\rangle \notin B^{\prime}$.

We will also need to find this inconsistency in $B^{\prime}$. For this we define the set $\hat{B}$ as follows: we add in all $\left\langle b, i^{\prime}, j^{\prime}, x\right\rangle$, where $\left\langle i^{\prime}, j^{\prime}\right\rangle$ is less than $\langle i, j\rangle$, where $\langle i, j\rangle$ is the location of the first inconsistency with $M_{K}$.

$$
\hat{B}=\left\{\left\langle b, i^{\prime}, j^{\prime}, x\right\rangle \mid\left\langle i^{\prime}, j^{\prime}\right\rangle \text { is less than the minimal }\langle i, j\rangle \text { with }\langle b, i, j, x\rangle \notin B^{\prime}\right\} .
$$


Notice that both $B^{\prime}$ and $\hat{B}$ are in EXP, therefore there are polynomial time reductions from $B^{\prime}$ and $\hat{B}$ to $A$ which use one bit of advice. Let $M_{B^{\prime}}^{b}$ and $M_{\hat{B}}^{b}$ be these reductions respectively.

We are now ready to define the polynomial time Turing machine with oracle access to $A$ which computes $x \in K$.

On input $x$

1. Compute $p(|x|)$ where $2^{p(|x|)}$ is the running time of $M_{K}$. (Recall that $M_{K}$ is the exponential machine computing $K$.)

2. Compute $q_{0}=M_{B}^{0}\left(10^{p(|x|)}, 10^{p(|x|)}, x\right)$ and $q_{1}=M_{B}^{1}\left(10^{p(|x|)}, 10^{p(|x|)}, x\right)$, where $M_{B}^{b}$ is the polynomial time reduction from $B$ to $A$ using advice $b$.

3. Ask $q_{0} \in A$ and $q_{1} \in A$

If both yes ACCEPT

Else if both no, REJECT

Else continue

4. Now we will assume that 0 was the correct advice and that $M_{B}^{0}\left(10^{p(|x|)}\right.$, $\left.10^{p(|x|)}, x\right) \in A$ is the right answer. This assumption also implies that there is some $\langle i, j\rangle$ such that $\langle 1, i, j, x\rangle \notin B^{\prime}$. We will use $\hat{B}$ to find such a location. We do this using the reduction $M_{\hat{B}}^{b}$. Compute $l_{0}=\max \langle i, j\rangle$ with $M_{\hat{B}}^{0}(\langle 1, i, j, x\rangle) \in A$ and $l_{1}=\max \langle i, j\rangle$ with $M_{\hat{B}}^{1}(\langle 1, i, j, x\rangle) \in A$.

5. There are three possibilities from above:

- If no $l_{0}$ or $l_{1}$ is found then there is no inconsistency with the 1 -tableau, i.e. 1 is the right advice. If $q_{1} \in A$ ACCEPT, else REJECT.

- One $l_{0}$ or $l_{1}$ is found or $l_{0}=l_{1}$. This means that there is an inconsistency at $l_{0}$ and 0 is the correct advice. If $q_{0} \in A$ ACCEPT, else REJECT.

- Both $l_{0}$ and $l_{1}$ are found and $l_{0} \neq l_{1}$. Here exactly one of $l_{0}, l_{1}$ must be correct and there is an inconsistency within the 1-tableau and 0 is the correct advice. If $q_{0} \in A$ ACCEPT, else REJECT.

With a slightly more complicated proof the above theorem can be extended to reductions using up to $c \log n$ advice. The different advice strings then give rise to a polynomial number of (possibly disagreeing) tableaux. With help of the one correct advice, an exponential time computation, and thus a polynomial time reduction, can point out the first errors in the other computations, thus providing proof for the correct result.

The tableau technique is a nonrelativizing technique. It is no surprise that this theorem does not relativize. We make this explicit in the following theorem.

Theorem 33 There exists an oracle $A$ and an set $B$ that is $\leq_{m}^{p / 1, A}$-complete for $\mathrm{EXP}^{A}$ but not $\leq_{T}^{p, A}$-complete for $\mathrm{EXP}^{A}$.

Proof For this proof we use the construction of an oracle appearing in [15]. Here, a set $A$ is constructed such that $\operatorname{EXP}^{A}$ has a Turing complete sets that is not autoreducible. In particular there is a subset of $S$ of $\left\{0^{n}\right\}$ such that $A-S$ is no longer 
Turing complete. The Turing reduction that makes $A$ complete is a very simple one. If $0^{|x|} \in A$ then $x \in K \leftrightarrow\langle 0, x\rangle \in A$ else $x \in K \leftrightarrow\langle 1, x\rangle \in A$. Of course this is a $\leq_{m}^{p / 1}$ reduction and $A-S$ remains $\leq_{m}^{p / 1}$ complete under this reduction

The fact that many-one completeness with one bit of advice can be simulated using more queries, extends to the delta levels of the polynomial hierarchy. Here the reduction does not even have to be adaptive as the following theorem shows.

Theorem 34 For every $k$ and every set $A$ in $\Delta_{k}^{p}$, if $A$ is $\leq_{m}^{p / 1}$-complete, then $A$ is $\leq_{\mathrm{tt}}^{p}$-complete.

Proof Let $X^{i}$ be the set of variables $x_{1}^{i}, x_{2}^{i}, \ldots, x_{n}^{i}$ and denote a particular assignment to $X^{i}$ as the string $\omega_{i}$ where the $j$ th bit is the value of $x_{j}^{i}$. For each level $k$ of $\Delta_{k}^{p}$, we fix $\phi$, a boolean formula over $X^{1} \cup X^{2} \cup \cdots \cup X^{k}$ with $k n$ variables. Consider the sets:

$S_{k}(\phi)=\left\{X^{1} \mid \forall X^{2} \exists X^{3} \forall \cdots Q X^{k} \phi\left(X^{1}, X^{2}, \ldots, X^{k}\right)\right.$ is true $\}$,

$L_{k}=\left\{\langle\phi, j\rangle \mid\right.$ the $j$ th bit in the lexicographically least element in $S_{k}(\phi)$ is 1$\}$,

$W_{k}(\phi, X)=\left\{0^{n} \mid \exists Y, \forall X^{1} \exists \cdots Q X^{k-2} \phi\left(X, Y, X^{1}, \ldots, X^{k-2}\right)\right.$ is not true $\}$.

Notice that $L_{k}$ is complete for $\Delta_{k}^{p}$ [26] and that $W_{k}(\phi, X) \in \Delta_{k-1}^{p}$. Let $A$ be a $\leq_{m}^{p / 1}$ complete set for $\Delta_{k}^{p}$. We show how to compute $L_{k}$ using a truth table reduction to $A$. We will do this by induction on the level $k$.

We first show this for $\mathrm{P}^{\mathrm{NP}}$ as a base case, namely, that $k=1$. Let $M_{1}$ compute the reduction from $L_{1}$ to $A$ given advice $b$, and let $M_{1}^{b}(\langle\phi, j\rangle)$ be the output of the reduction, i.e., for the correct advice $b$ it holds that $M_{1}^{b}(\langle\phi, j\rangle) \in A$ if and only if $\langle\phi, j\rangle \in L_{1}$. We pad $L_{1}$, such that $\langle\phi, 0\rangle, \ldots,\langle\phi, n\rangle$ are all of the same length, i.e., use the same advice.

Now compute

$$
\begin{aligned}
& \omega_{1}^{0}=\left\langle M_{1}^{0}(\langle\phi, 0\rangle)\right\rangle, \ldots,\left\langle M_{1}^{0}(\langle\phi, n\rangle)\right\rangle, \\
& \omega_{1}^{1}=\left\langle M_{1}^{1}(\langle\phi, 0\rangle)\right\rangle, \ldots,\left\langle M_{1}^{1}(\langle\phi, n\rangle)\right\rangle .
\end{aligned}
$$

We know at least one of $\phi\left(\omega_{1}^{0}\right)$ or $\phi\left(\omega_{1}^{1}\right)$ must be true as 0 or 1 must be the correct advice and return the bits of the lexicographically least assignment to $\phi$. This implies that the other advice either returns an assignment that does not make $\phi$ true, or an assignment that is not lexicographically smaller. In any case, by asking the queries $M_{1}^{0}(\langle\phi, 0\rangle), \ldots, M_{1}^{0}(\langle\phi, n\rangle)$ and $M_{1}^{1}(\langle\phi, 0\rangle), \ldots, M_{1}^{1}(\langle\phi, n\rangle)$ we can check which is which in polynomial time. Thus giving us a $\leq_{\mathrm{tt}}^{p}$ reduction to $A$ and completing our base case.

Now we assume that there is a $\leq_{\mathrm{tt}}^{p}$-reduction from $L_{k-1}$ to A. As before, let $M_{k}$ compute the $\leq_{m}^{p / 1}$-reduction from $L_{k}$ to $A$ and let $M_{k}^{b}(\langle\phi, j\rangle)$ be the output for the correct advice $b$. Again compute:

$$
\begin{aligned}
& \omega_{k}^{0}=\left\langle M_{k}^{0}(\langle\phi, 0\rangle)\right\rangle, \ldots,\left\langle M_{k}^{0}(\langle\phi, n\rangle)\right\rangle, \\
& \omega_{k}^{1}=\left\langle M_{k}^{1}(\langle\phi, 0\rangle)\right\rangle, \ldots,\left\langle M_{k}^{1}(\langle\phi, n\rangle)\right\rangle .
\end{aligned}
$$


We know that one of $\phi\left(\omega_{k}^{0}\right)$ or $\phi\left(\omega_{k}^{1}\right)$ is an element of $S_{k}(\phi)$. To determine which we use the fact that $L_{k-1}$ is $\Delta_{k-1}^{p}$ complete and the $\leq_{\mathrm{tt}}^{p}$-reduction from $W_{k}\left(\phi, \omega_{k}^{0}\right)$ to $A$ and the inductive assumption that there is a $\leq_{\mathrm{tt}}^{p}$-reduction from $W_{k}\left(\phi, \omega_{k}^{1}\right)$ to $A$. We ask if $0^{\left|\omega_{k}^{0}\right|} \in W_{k}\left(\phi, \omega_{k}^{0}\right)$ and $0^{\left|\omega_{k}^{1}\right|} \in W_{k}\left(\phi, \omega_{k}^{1}\right)$. At least one of these queries will be no as 0 or 1 is the correct advice. If only one says no, we have determined the correct advice and have a $\leq_{\mathrm{tt}}^{p}$-reduction. If the both are no, one of the assignments is lexicographically smaller and we again know the correct advice and have a $\leq_{\mathrm{tt}^{-}}^{p}$ reduction.

Theorem 35 [15] EXPSPACE has a set that is complete using a many-one reduction with one bit of advice and is not complete using any truth table reduction.

Proof Consider the EXPSPACE-complete set created by Buhrman et al. [15] which is complete under truth table reductions that ask 3 queries, but is not autoreducible under nonadaptive reductions. This set $A$ is a subset of $\{0\}^{*} \cup\langle 0, x\rangle \cup\langle 1, x\rangle$. The set has the property that $x \in K \leftrightarrow 0^{|x|} \in A \wedge\langle 0, x\rangle \in A$ or $x \in K \leftrightarrow 0^{|x|} \notin A \wedge\langle 1, x\rangle \in A$. Here, $K$ is the canonical EXPSPACE complete set. The nonadaptive reduction on input $x$ queries $0^{|x|},\langle 0, x\rangle,\langle 1, x\rangle$. To show that the set is not autoreducible they construct the set so that all nonadaptive reductions must query $0^{|x|}$ to be complete.

We use this fact for our claim, consider the set $A-\{0\}^{*}$ from [15] we know that this set is not complete under nonadaptive reductions. However, consider the manyone reduction $x \in K \rightarrow\langle b, x\rangle$, where $b$ is the advice bit, and $b=0 \leftrightarrow 0^{|x|} \in A$. Here it is clear that $K \leq_{m}^{p / 1} A-\{0\}^{*}$. This yields a set in EXPSACE complete under manyone reductions which use one bit of advice that is not complete under nonadaptive reductions.

The two previous theorems have some interesting consequences. We don't know whether Theorem 32 can be strengthened to truth-table reductions. However there are two cases.

1. By Theorem 35, if $\leq_{m}^{p / 1}$-completeness implies $\leq_{\mathrm{tt}}^{p}$-completeness on EXP then $\mathrm{EXP} \neq \mathrm{EXPSPACE}$.

2. By Theorem 34, we know if $\leq_{m}^{p / 1}$-completeness does not imply $\leq_{\mathrm{tt}}^{p}$-completeness on $\mathrm{EXP}$, then $\mathrm{PH} \neq \mathrm{EXP}$.

\section{Further Research}

We have initiated the systematic study of non-uniform reductions. Many of the results here concern reductions which use only 1 bit of advice. In Theorem 19, we do not know that even this one bit of advice in necessary. Can it be eliminated? In places where more advice bits are needed can they be reduced? In general, how much stronger are non-uniform reductions than their uniform counterparts.

Another, more technical question about reductions left open here, is whether the extended many-one reductions used in Theorem 2 are really needed? This may be 
more difficult than it first seems as Theorem 13 presents an oracle where length increasing many-one reductions do not exist for NP-complete sets. Can we add an immunity assumption to this oracle showing that non-relativizing techniques would be required to prove Theorem 2 for standard many-one reductions.

Another line of research is to strengthen some of the theorems concerning NP by weakening the strong hypotheses used in their proof. In particular, are the immunity and measure assumptions necessary for Theorems 2 and 19? Part of this paper was motivated by the recent work of Hitchcock and Pavan [21]. That paper features and compares a number of these strong hypotheses, and it would be interesting to determine the minimal hypotheses needed in our results here.

Acknowledgements We thank John Hitchcock, Eric Allender, and Stephen Fenner for useful discussions and feedback. We thank John Hitchcock for his observation that the hypothesis in our length increasing result for NP can be weakened to bi-immunity instead of measure zero.

Open Access This article is distributed under the terms of the Creative Commons Attribution Noncommercial License which permits any noncommercial use, distribution, and reproduction in any medium, provided the original author(s) and source are credited.

\section{References}

1. Agrawal, M.: Pseudo-random generators and structure of complete degrees. In IEEE Conference on Computational Complexity, pp. 139-147 (2002)

2. Agrawal, M., Biswas, S.: Polynomial isomorphism of 1-L complete sets. In: Proc. Structure in Complexity Theory 7th Annual Conference, San Diego, California, pp. 75-80. IEEE Computer Society, Los Alamitos (1993)

3. Allender, E.: Isomorphisms and 1-L reductions. J. Comput. Syst. Sci. 36(6), 336-350 (1988)

4. Allender, E., Buhrman, H., Koucký, M., van Melkebeek, D., Ronneburger, D.: Power from random strings. In: FOCS, pp. 669-678. IEEE Computer Society, Los Alamitos (2002)

5. Ambos-Spies, K.: p-mitotic sets. In: Börger, E., Hasenjäger, G., Roding, D. (eds.) Logic and Machines. Lecture Notes in Computer Science, vol. 177, pp. 1-23. Springer, Berlin (1984)

6. Balcázar, J., Díaz, J., Gabarró, J.: Structural Complexity I. Springer, Berlin (1988)

7. Berman, L., Hartmanis, H.: On isomorphisms and density of NP and other complete sets. SIAM J. Comput. 6, 305-322 (1977)

8. Buhrman, H., Mayordomo, E.: An excursion to the Kolmogorov random strings. In: Proceedings Structure in Complexity Theory, 10th Annual Conference (STRUCTURES95), Minneapolis, pp. 197205. IEEE Computer Society, Los Alamitos (1995)

9. Buhrman, H., Torenvliet, L.: Complicated complementations. In: Proceedings 14th IEE Conference on Computational Complexity, pp. 227-236. IEEE Computer Society, Los Alamitos (1999)

10. Buhrman, H., Torenvliet, L.: Separating complexity classes using structural properties. In: Proceedings 19th IEE Conference on Computational Complexity, pp. 130-138. IEEE Computer Society, Los Alamitos (2004)

11. Buhrman, H., Torenvliet, L.: A Post's program for complexity theory. In Bulletin of the EATCS 85, pp. 41-51 (2005)

12. Buhrman, H., Homer, S., Torenvliet, L.: On complete sets for nondeterministic classes. Math. Syst. Theory 24, 179-200 (1991)

13. Buhrman, H., Spaan, E., Torenvliet, L.: Bounded reductions. In: Ambos-Spies, K., Homer, S., Schöning, U. (eds.) Complexity Theory, pp. 83-99. Cambridge University Press, Cambridge (1993)

14. Buhrman, H., Spaan, E., Torenvliet, L.: The relative power of logspace and polynomial time reductions. Comput. Complexity 3(3), 231-244 (1993)

15. Buhrman, H., van Melkebeek, D., Fortnow, L., Torenvliet, L.: Using autoreducibility to separate complexity classes. SIAM J. Comput. 29(5), 1497-1520 (2000)

16. Fenner, S., Fortnow, L., Kurtz, S.A.: The isomorphism conjecture holds relative to an oracle. In: Proc. 33rd IEEE Symposium Foundations of Computer Science, pp. 30-39 (1992) 
17. Ganesan, K., Homer, S.: Complete problems and strong polynomial reducibilities. In: Proc. Symposium on Theoretical Aspects of Computer Science. Springer Lecture Notes in Computer Science, vol. 349, pp. 240-250. Springer, Berlin (1988)

18. Glaßer, C., Selman, A.L., Travers, S.D., Zhang, L.: Non-mitotic sets. In: Arvind, V., Prasad, S. (eds.) STTCS. Lecture Notes in Computer Science, vol. 4855, pp. 146-157. Springer, Berlin (2007)

19. Hartmanis, J., Hemachandra, L.: One-way functions and the non-isomorphism of NP-complete sets. Theor. Comput. Sci. 81(1), 155-163 (1991)

20. Homer, S., Kurtz, S., Royer, J.: A note on many-one and 1-truth table complete sets. Theor. Comput. Sci. 115(2), 383-389 (1993)

21. Hitchcock, J.M., Pavan, A.: Hardness hypotheses, derandomization, and circuit complexity. In: 24th Conference on Foundations of Software Technology and Theoretical Computer Science, pp. 336-347. Springer, Berlin (2004)

22. Hitchcock, J.M., Pavan, A.: Comparing reductions to NP-complete sets. In: Bugliesi, M., Preneel, B., Sassone, V., Wegener, I. (eds.) ICALP (1). Lecture Notes in Computer Science, vol. 4051, pp. 465-476. Springer, Berlin (2006)

23. Homer, S., Selman, A.L.: Oracles for structural properties: the isomorphism problem and public-key cryptography. J. Comput. Syst. Sci. 44(2), 287-301 (1992)

24. Homer, S., Selman, A.L.: Computability and Complexity Theory. Springer, New York (2001)

25. Kurtz, S., Mahaney, S., Royer, J.: The isomorphism conjecture fails relative to a random oracle. In Proc. 21nd Annual ACM Symposium on Theory of Computing, pp. 157-166 (1989)

26. Krentel, M.: The complexity of optimization problem. J. Comput. Syst. Sci. 36, 490-509 (1988)

27. Ladner, R., Lynch, N., Selman, A.: A comparison of polynomial time reducibilities. Theor. Comput. Sci. 1, 103-123 (1975)

28. Mayordomo, E.: Almost every set in exponential time is p-bi-immune. Theor. Comput. Sci. 136(2), 487-506 (1994)

29. Ronneburger, D.: Kolmogorov complexity and derandomization. PhD thesis, Rutgers University, New Brunswick, NJ, October 2004

30. Watanabe, O.: A comparison of polynomial time completeness notions. Theor. Comput. Sci. 54, 249265 (1987)

31. Young, P.: Juris Hartmanis: Fundamental contributions to the isomorphism problems. In: Selman, A.L. (ed.) Complexity Theory Retrospective, pp. 108-146. Springer, Berlin (1990) 